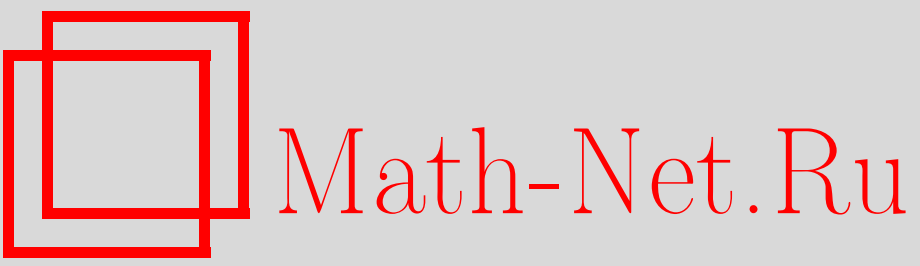

А. Н. Васильев, М. И. Вязовский, С. Э. Деркачев, Н. А. Кивель, Об эквивалентности ренормировок в обычной и размерной регуляризациях для $2 D$ четырехфермионных взаимодействий, ТМФ, 1996, том 107, номер 1, 27-46

DOI: https://doi.org/10.4213/tmf1136

Использование Общероссийского математического портала Math-Net.Ru подразумевает, что вы прочитали и согласны с пользовательским соглашением

http://www.mathnet.ru/rus/agreement

Параметры загрузки:

IP : 54.147 .182 .235

26 апреля 2023 г., 17:08:04 


\author{
ТЕОРЕТИЧЕСКАЯ \\ И МАТЕМАТИЧЕСКАЯ \\ ФИЗИКА \\ Том 107, № 1 \\ апрель, 1996
}

А.Н. Васильев, М.И. Вязовский, С.Э. Деркачев, Н.А. Кивель

\title{
ОБ ЭКВИВАЛЕНТНОСТИ РЕНОРМИРОВОК В ОБЫЧНОЙ И РАЗМЕРНОЙ РЕГУЛЯРИЗАЦИЯХ ДЛЯ 2D-ЧЕТЫРЕХФЕРМИОННЫХ ВЗАИМОДЕЙСТВИЙ
}

Рассматривается проблема эквивалентности ренормировок полной $U_{N}$-симметричной 4F-модели [1] с обычной (без выхода из целой размерности $d=2$ ) и размерной $d=2+\varepsilon$ регуляризациями. В первом случае теория трехзарядна, во втором для мультипликативной ренормируемости необходимо ввести бесконечное число независимых зарядов $g \equiv\left\{g_{n}, n=0,1, \ldots\right\}$. Показано, что после обычной MS-ренормировки размерно регуляризованной модели можно выполнить такую УФ-конечную ренормировку полей и зарядов $g \rightarrow g^{\prime}(g)$, после которой ренормированные функции Грина в пределе $\varepsilon \rightarrow 0$ будут зависеть уже не от всех, а только от трех младших зарядов $g_{n}^{\prime}(g)$ с $n=0,1,2$, чем и обеспечивается возможность эквивалентности разных ренормировочных схем. Приводятся результаты расчета в схеме MS с двухпетлевой точностью для $\beta$-функций и с трехпетлевой для аномальной размерности поля $\gamma_{\psi}$, а также вывод соотношений "проекционной техники" $[1,2]$, позволяющей выразить при $\varepsilon=0$ старшие ренормированные составные операторы $4 \mathrm{~F}$-взаимодействия через младшие.

\section{1. ВВЕДЕНИЕ}

Одним из символов веры теории мультипликативной ренормировки является утверждение о физической эквивалентности любых двух ренормированных теорий, получаемых разными процедурами регуляризации и (или) ренормировки из одной и той же неренормированной. Физическая эквивалентность означает, что соответствующие ренормированнные функции Грина связаны между собой УФ-конечной мультипликативной ренормировкой полей и параметров: параметры второй (ренормированные массы, заряды и т.п.) являются некоторыми У $\Phi$-конечными функциями параметров первой, а соответствующие ренормированные операторы поля $\hat{\phi}_{\mathrm{R}}$ и $\hat{\phi}_{\mathrm{R}}^{\prime}$ различаются только УФ-конечным множителем: $\hat{\phi}_{R}=Z_{\phi}^{\prime} \hat{\phi}_{R}^{\prime}$.

Не сомневаясь в справедливости обшего утверждения об эквивалентности, мы хотим в данной работе обратить внимание на то, что конкретная его реализация оказывается весьма нетривиальной для двумерных (2D) четырехфермионных (4F) моделей при сопоставлении их ренормировки в обычной (без выхода из размерности $d=2$ ) и размерной $d=2+\varepsilon$ регуляризациях. Дело в том, что в целой размерности алгебра $\gamma$-матриц конечномерна, и поэтому сушествует лишь конечное число независимых 4 F-взаимодействий. 
В нецелой размерности можно пользоваться только стандартным коммутационным соотношением $\gamma_{i} \gamma_{k}+\gamma_{k} \gamma_{i}=2 \delta_{i k}$ (мы будем рассматривать евклидов вариант), позволяющим привести любое произведение $\gamma$-матриц к линейной комбинации полностью антисимметризованных произведений

$$
\gamma_{A}^{(n)} \equiv \operatorname{As}\left[\gamma_{i_{1}} \cdot \ldots \cdot \gamma_{i_{n}}\right], \quad \mathrm{A} \equiv\left\{i_{1}, \ldots, i_{n}\right\}
$$

В нецелой размерности все эти объекты приходится считать отличными от нуля независимыми структурами [3], образующими полный базис в пространстве действующих на спиноры $\psi$ матриц. Каждой структуре (1) сопоставляется независимое локальное 4F-взаимодействие

$$
V_{n} \equiv \frac{1}{2} \bar{\psi} \gamma_{A}^{(n)} \psi \cdot \bar{\psi} \gamma_{A}^{(n)} \psi
$$

(по векторным индексам А - суммирование, нормировка $\frac{1}{2}$ - для удобства), и при расчетах в любой 4F-модели с размерной регуляризацией старшие взаимодействия (2) будут появляться в качестве контрчленов.

Поэтому мультипликативная ренормируемость (необходимая для стандартного доказательства физической эквивалентности) в размерной регуляризации будет гарантирована только тогда, когда в неренормированное действие с самого начала включаются все взаимодействия (2) с независимыми коэффициентами - затравочными зарядами $g_{0} \equiv\left\{g_{0 n}, n=0,1,2, \ldots\right\}$, а соответствующая ренормированная (например, в схеме MS) теория будет содержать бесконечное число независимых ренормированных зарядов $g \equiv\left\{g_{n}, n=0,1,2, \ldots\right\}$.

В целой размерности $d$ отличны от нуля лишш величины (1) с $n \leq d$. Такие структуры, а также взаимодействия (2) и соответствующие заряды будем в дальнейшем называть "младшими", а объекты с $n>d$ - "старшими" ("evanescent operators" [1]). Bce $4 \mathrm{~F}$-взаимодействия (2) логарифмичны в размерности $d=2$ (это та размерность, в которой все вершинные диаграммы расходятся логарифмически), младшими для нее являются лишь вершины с $n=0,1,2$ ( $S, V, P$ в других обозначениях). Непосредственно для двухкомпонентных спиноров эти взаимодействия линейно зависимы в силу двумерных соотношений Фирца $\left(2 V_{0}=V_{2}=-V_{1}\right)$. Поэтому мы будем рассматривать для обшности $U_{N}$-симметричную модель [1] с дополнительным “изотопическим" индексом $a=1,2, \ldots, N$ у спиноров $\bar{\psi}, \psi$ и подразумеваемым суммированием по нему во всех квадратичных формах $\bar{\psi} \ldots \psi$, в которой три младшие взаимодействия независимы. Неренормированное действие полей $\phi \equiv(\bar{\psi}, \psi)$ безмассовой $U_{N}$-симметричной $4 \mathrm{~F}$-модели с размерной регуляризацией будем записывать в виде

$$
S(\phi)=\bar{\psi} \hat{\partial} \psi+\sum_{n=0}^{\infty} g_{0 n} V_{n}
$$

подразумевая все нужные интегрирования и суммирования по индексам, а также запись $\exp S$ в евклидовом функциональном интеграле без обычного минуса в показателе.

В любой обычной (без выхода из целой размерности $d=2$ ) регуляризации в $(3)$ отличны от нуля лишь вклады трех младших взаимодействий, и соответствуюшая ренормированная теория трехзарядна. В размерной регуляризации число независимых зарядов бесконечно; после устранения ренормировкой (например, в схеме MS) полюсов по $\varepsilon$ 
и перехода к пределу $\varepsilon=0$ мы получим ренормированные функции Грина, зависяшие от бесконечного числа ренормированных зарядов $g$. Поэтому возникает естественный вопрос: каким образом могут быть эквивалентны ренормированные теории с разным (три в одном случае и $\infty$ в другом) числом ренормированных зарядов?

В данной статье мы хотим предложить простой ответ на этот вопрос. Суть состоит в следуюшем: эквивалентность возможна, если функции бесконечного числа переменных $g$ зависят от них в действительности только через посредство конечного числа некоторых других величин $g_{n}^{\prime}(g), n=0,1,2$, которых ровно столько, сколько младших зарядов (три). Мы покажем, что именно так и реализуется эквивалентность между ренормированными теориями с обычной и размерной регуляризациями. Более точно, будет показано, что для теории с размерной регуляризацией сушествует такая отличная от MS схема вычитаний (или $R$-операция), для которой соответствующие ренормированные функции Грина в пределе $\varepsilon=0$ будут зависеть только от ее младших зарядов $g_{n}^{\prime}$, $n=0,1,2$, и отличаться от ренормированных функций схемы MS лишш УФ-конечной ренормировкой.

Точная формулировка состоит в следующем: пусть $\Gamma\left(\phi, g_{0}\right)$ - производящий функционал неренормированных 1-неприводимых функций Грина модели (3) с бесконечным числом зарядов в размерности $d=2+2 \varepsilon, \Gamma_{\mathrm{R}}^{\mathrm{MS}}(\phi, g)=\Gamma\left(Z_{\phi} \phi, g_{0}\right)$ - аналогичный функционал для ренормированных в стандартной схеме MS функций Грина с ренормированными зарядами $g=g\left(g_{0}\right)$.

УТВЕРЖДЕНИЕ. Существует УФ-конечная ренормировка $g \rightarrow g^{\prime}(g), \phi \rightarrow Z_{\phi}^{\prime} \phi$, такая, что функиионал

$$
\Gamma_{\mathrm{R}}^{\prime}\left(\phi, g^{\prime}\right)=\Gamma_{\mathrm{R}}^{\mathrm{MS}}\left(Z_{\phi}^{\prime} \phi, g\right), \quad g=g\left(g^{\prime}\right), \quad Z_{\phi}^{\prime}=Z_{\phi}^{\prime}(g)
$$

в пределе $\varepsilon=0$ зависит только от младиих зарядов $g_{n}^{\prime}$ с $n=0,1,2$.

В (4) подразумевается, хотя и не указывается явно, зависимость от параметра регуляризации $\varepsilon$ и ренормировочной массы $\mu$. Отметим также, что конечная ренормировка типа (4), естественно, неоднозначна: любая последующая замена $\phi \rightarrow Z_{\phi}^{\prime \prime} \phi, g^{\prime} \rightarrow g^{\prime \prime}$, при которой $g^{\prime \prime}$ и $Z_{\phi}^{\prime \prime}$ зависят только от младших зарядов $g^{\prime}$, также будет обладать нужными свойствами. Преобразования такого типа и будут связывать получаемые с помощью процедуры (4) при $\varepsilon=0$ функции Грина с теми, которые получаются с помощью обычной (без выхода из целой размерности $d=2$ ) регуляризации и ренормировки.

Проблема исключения старших зарядов в модели (3) уже исследовалась в работе [1], но только применительно к $\beta$-функциям младших зарядов при $\varepsilon=0$. В данной работе доказывается наличие нужного свойства непосредственно для функций Грина, что автоматически влечет аналогичное свойство как для $\beta$-функций младших зарядов, так и для аномальной размерности поля $\gamma_{\psi}$, которую мы вычислили с трехпетлевой точностью (п. 3). Кроме того, будет показано (п. 4), что из утверждения (4) легко получаются формулы “проекционной техники" $[1,2]$, позволяюшие выразить при $\varepsilon=0$ старшие ренормированные составные операторы взаимодействия (2) через три младших с явным выражением всех коэффициентов через характеризуюшие УФ-конечную ренормировку функции $Z_{\phi}^{\prime}(g), g^{\prime}(g)$ в $(4)$. 


\section{2. ПРОВЕРКА УТВЕРЖДЕНИЯ (4) В НИЗШИХ ПОРЯДКАХ}

В этом разделе мы поясним утверждение (4) и проверим его справедливость в низшем нетривиальном порядке теории возмушений (одна петля в вершине $\Gamma_{4}$ и две в собственной энергии $\Gamma_{2}$ ), а в п. 3,4 рассмотрим следующие порядки и приведем общее доказательство.

Предварительно условимся об обозначениях: при нумерации структур (1), вершин (2) и соответствуюших зарядов будем использовать буквы $k, m, n, s, l$ для обозначения номеров, принимающих все значения от 0 до $\infty$, буквы $a, b, c$ - только для младших номеров $0,1,2$, а буквы $\alpha, \beta, \lambda$ - только для старших $k>2$. Всюду в дальнейшем будут также использоваться обозначения

$$
u_{k} \equiv g_{k} / 4 \pi, \quad n \equiv N \operatorname{tr} 1, \quad d=2+2 \varepsilon
$$

Деление всех зарядов на $4 \pi$ упрошает вид РГ-функций, устраняя из них все множители $\pi$. Множитель $n=N \operatorname{tr} 1$ порождается замкнутыми циклами фермионных линий, $N-$ целое число изотопических компонент, $\operatorname{tr} 1$ - след единичной операции в пространстве $d$-мерных спиноров, не имеющий явного выражения через $d$ в нецелой размерности (при $d=2$ считаем $\operatorname{tr} 1=2$ ). Первые коэффициенты разложения по $\varepsilon$ любой регулярной по $\varepsilon$ величины $F(\varepsilon)$ будут обозначаться следуюшим образом:

$$
F=\bar{F}+\varepsilon \overline{\bar{F}}+\ldots
$$

Соответствуюшее неренормированной теории (3) базовое действие $S_{\text {баз. }}$ (к диаграммам которого применяется $R$-операция) и ренормированное действие $S_{\mathrm{R}}$ имеют в тех же обозначениях вид

$$
\begin{aligned}
S_{\text {баз. }}(\phi) & =\bar{\psi} \hat{\partial} \psi+\mu^{-2 \varepsilon} \sum_{n=0}^{\infty} g_{n} V_{n}, \\
S_{\mathrm{R}}(\phi) & =Z_{1} \bar{\psi} \hat{\partial} \psi+\mu^{-2 \varepsilon} \sum_{n=0}^{\infty} Z_{2 n} g_{n} V_{n},
\end{aligned}
$$

где $\mu$ - ренормировочная масса, а $g \equiv\left\{g_{n}\right\}$ - бесконечный набор ренормированных зарядов. Действие (8) получается из (3) стандартной процедурой мультипликативной ренормировки (в записи переходим к обозначениям (5)):

$$
\begin{gathered}
S_{\mathrm{R}}(\phi ; u, \mu)=S\left(Z_{\phi} ; u_{0}(u, \mu)\right), \quad u_{0 k}=\mu^{-2 \varepsilon} u_{k} Z_{u_{k}} \\
Z_{\phi} \equiv Z_{\bar{\psi}}=Z_{\psi}=Z_{1}^{1 / 2}, \quad Z_{u_{k}}=Z_{2 k} Z_{1}^{-2}
\end{gathered}
$$

Ренормгрупповые (РГ) функции $\gamma$ аномальных размерностей поля $\psi$ и зарядов $u_{k}$ и $\beta$-функции этих зарядов $\beta_{k}$ определяются стандартными соотношениями:

$$
\gamma_{\psi}=\tilde{\mathcal{D}}_{\mu} \ln Z_{\psi}, \quad \gamma_{u_{k}}=\tilde{\mathcal{D}}_{\mu} \ln Z_{u_{k}}, \quad \beta_{k}=\tilde{\mathcal{D}}_{\mu} u_{k}=u_{k}\left(2 \varepsilon-\gamma_{u_{k}}\right)
$$

где $\tilde{\mathcal{D}}_{\mu} \equiv \mu \partial_{\mu}$ при фиксированных затравочных $u_{0} \sim g_{0}$. В обычной схеме MS все константы $Z$ имеют вид $1+$ полюсы по $\varepsilon$, тогда все аномальные размерности $\gamma$ в $(10)$ от 
$\varepsilon$ не зависят, а в $\beta$-функциях зависимость от $\varepsilon$ присутствует лиш в беспетлевых вкладах $2 \varepsilon u_{k}$. В других схемах вычитаний, рассматриваемых ниже, эти простые свойства РГ-функций теряются. РГ-уравнения для ренормированных 1-неприводимых функций $\Gamma_{n \mathrm{R}}$ с $n$ полями $\phi=\psi, \bar{\psi}$ имеют следующий вид:

$$
\left[\mathcal{D}_{\mathrm{RG}}-n \gamma_{\psi}\right] \Gamma_{n \mathrm{R}}=0, \quad \mathcal{D}_{\mathrm{RG}}=\mu \frac{\partial}{\partial \mu}+\sum_{k=0}^{\infty} \beta_{k} \frac{\partial}{\partial u_{k}}
$$

где $\mathcal{D}_{\mathrm{RG}}$ есть оператор $\tilde{\mathcal{D}}_{\mu}$ в ренормированных переменных.
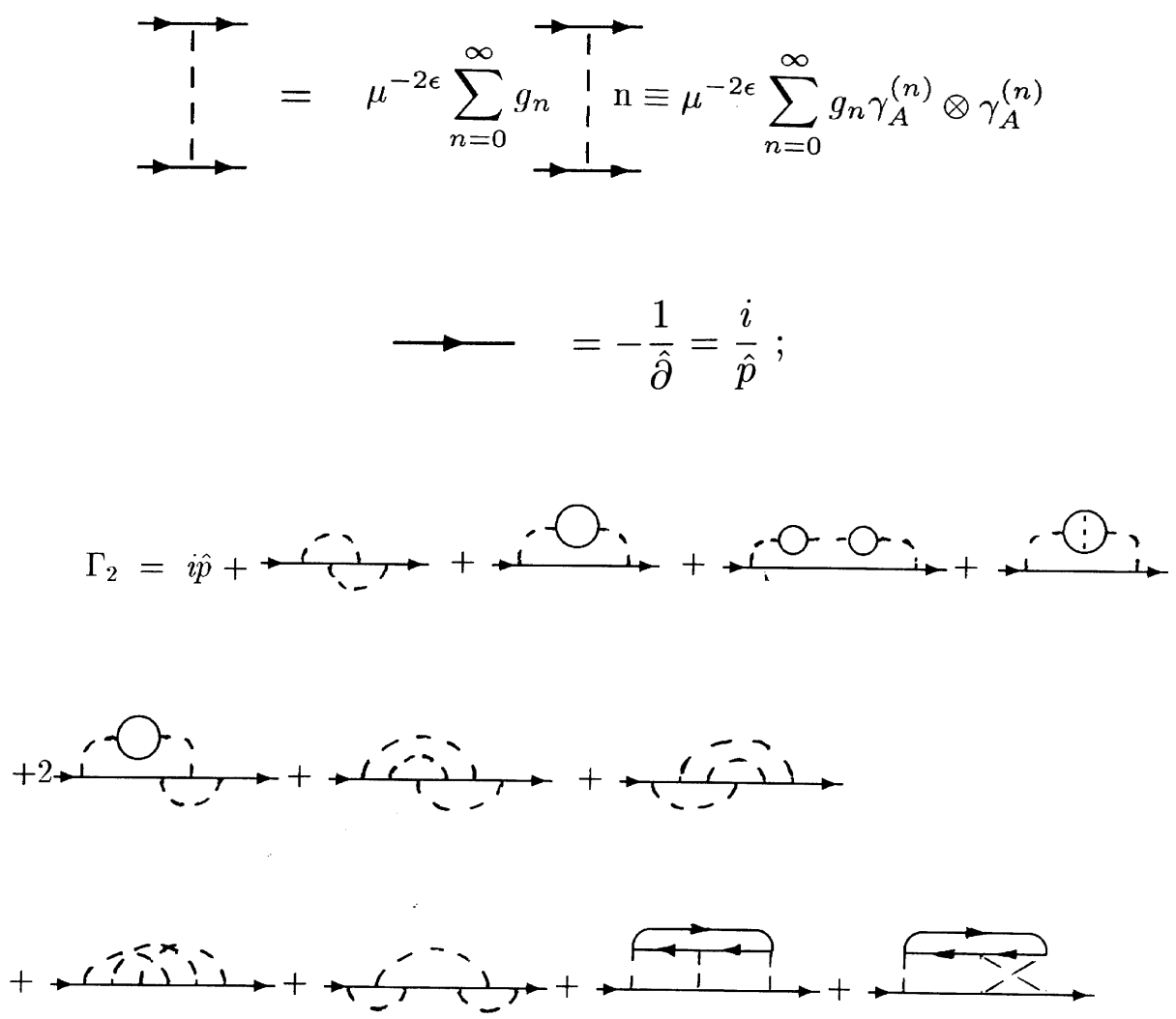

Для проверки утверждения (4) достаточно рассмотреть лишь содержашие поверхностные расходимости функции $\Gamma_{2,4}$ базовой теории (7). На рисунке приведены их линии и вершины и нужные для дальнейшего диаграммы $\Gamma_{2,4}$ вместе с беспетлевыми вкладами. В этом разделе мы ограничимся анализом первых поправок - однопетлевых диаграмм $\Gamma_{4}$ и двухпетлевых $\Gamma_{2}$ (в безмассовой теории с размерной регуляризацией однопетлевые диаграммы $\Gamma_{2}$ исчезают), в которых присутствуют лишш простые полюсы $\sim 1 / \varepsilon$. 


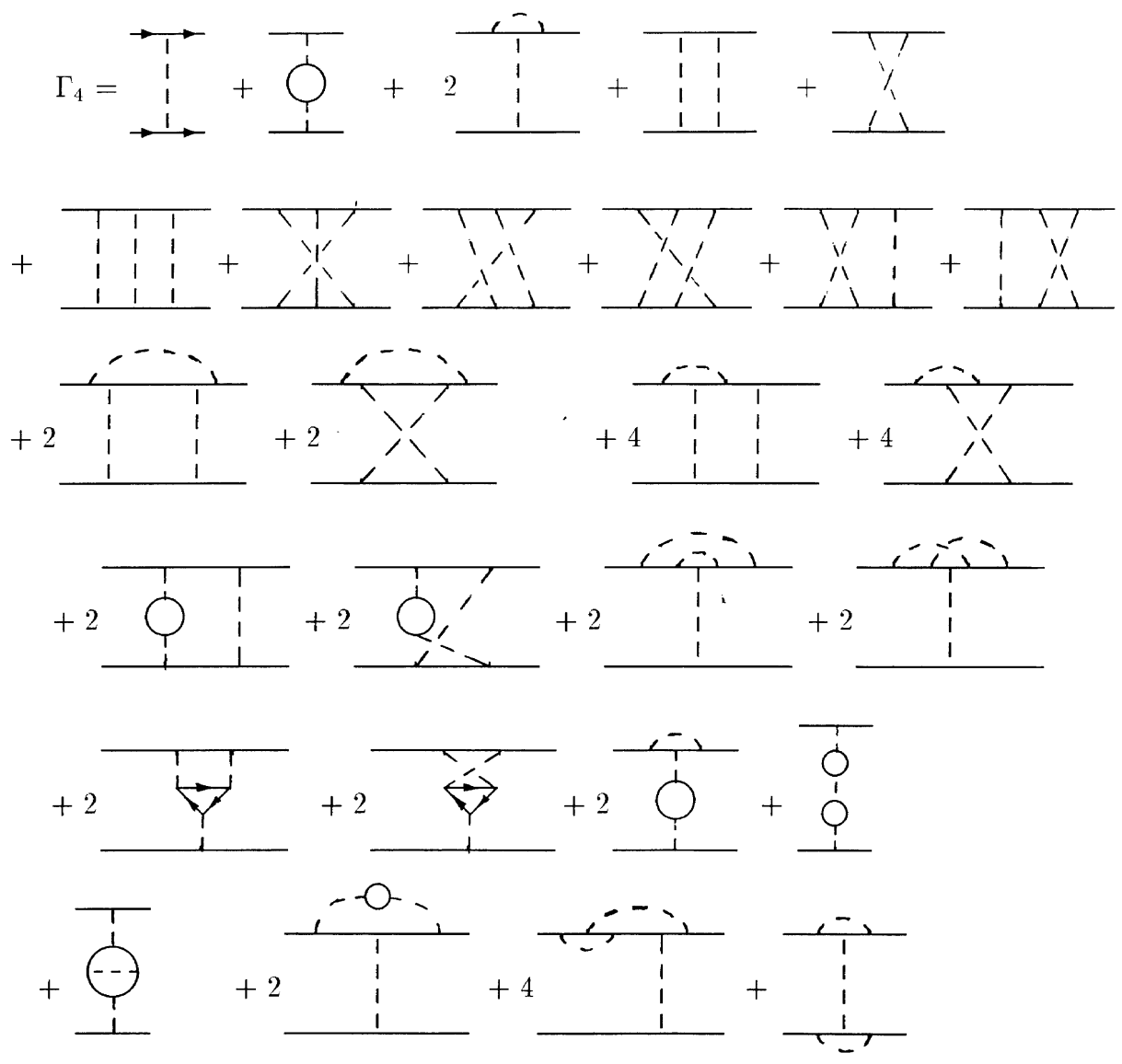

Вклад произвольной диаграммы $D$ представляется в виде произведения $D_{\gamma} D_{p}$ некоторой “ $\gamma$-структуры" $D_{\gamma}$ (к ней будем относить множители $\gamma$, в том числе и стоящие под знаком $\mathrm{tr}$ ) и "импульсного интеграла" $D_{p}$ с различными векторными индексами, которые сворачиваются между собой. Множитель $D_{\gamma}$ по $\varepsilon$ регулярен, а $D_{p}$ содержит УФ-расходимости - полюсы по $\varepsilon$. Их структура определяется обшими правилами теории ренормировки в размерной регуляризации: после вычитания стандартной $R^{\prime}$-операцией из импульсного интеграла $D_{p}$ всех его подрасходимостей получается выражение, расходяшаяся часть которого - полином по внешним импульсам $p$ нужной размерности. Для диаграмм $\Gamma_{2}$ это полином первого порядка по $p$, а для вершинных диаграмм $\Gamma_{4}$ - нулевого, т.е. выражение, составленное только из $\delta$-символов по векторным индексам. После вычисления следов и сворачивания по индексам получим "конечные $\gamma$-структуры" $D_{\gamma}$. Для диаграмм $\Gamma_{2}$ в произвольной размерности $d$ все они сводятся к $\hat{p}$, а для диаграмм $\Gamma_{4}$ роль полного базиса конечных $\gamma$-структур играют тензорные произведения $\gamma_{A}^{(k)} \otimes \gamma_{B}^{(m)}$ всех величин (1), в том числе и старших. После устранения ренормировкой полюсов по $\varepsilon$ и перехода к пределу $\varepsilon=0$ в конечных ответах могут оставаться только младшие $\gamma$-структуры, поскольку все старшие при $\varepsilon=0$ исчезают. По той же причине очевидно следуюшее утверждение: если исходное выражение для $D_{\gamma}$ 
содержит хотя бы один старший сомножитель (1), то при $\varepsilon=0$ имеем $D_{\gamma}=0$, поэтому в конечном выражении для $D_{\gamma}$ должны обрашаться в нуль коэффициенты при всех младших $\gamma$-структурах ( к старшим это не относится, поскольку они исчезают сами по себе). Это утверждение является основным для дальнейшего и для удобства ссылок сформулируем его в виде отдельного правила.

ПравИЛо 1. Вклад в $D_{\gamma}$ от стариих $\gamma$-структур в младшие при $\varepsilon=0$ равен нулю.

Вернемся теперь к диаграммам $\Gamma_{2,4}$ в нашем приближении (одна петля в $\Gamma_{4}$ и две в $\left.\Gamma_{2}\right)$. Их импульсные интегралы $D_{p}$ содержат регулярную по $\varepsilon$ У $\Phi$-конечную часть и локальные слагаемые с простым полюсом $\sim ~ 1 / \varepsilon$, соответствующие вклады будем обозначать через $A$ и $B$. Мы хотим понять, какой след оставляют старшие взаимодействия в ренормированных функциях $\Gamma_{n \mathrm{R}}$ при $\varepsilon^{\sim}=0$. Ясно, что в УФ-конечных вкладах типа $A$ можно просто положить $\varepsilon^{\sim}=0$, и в них тогда останется зависимость только от младших зарядов ввиду исчезновения старших взаимодействий. Поэтому для нас существенны лиш полюсные по $\varepsilon$ вклады типа $B$. Мы не приводим их расчет, так как он уже был выполнен ранее в работе [1] (две петли в $\Gamma_{2}$, одна и две в $\Gamma_{4}$ ) в рамках массивной модели (7). Мы перепроверили результаты [1] по диаграммам безмассовой модели (7) с простой протечкой одного внешнего импульса (вычисляемые величины от массы не зависят). Результаты совпали (отметим только, что в обозначенных через $D, G$ и $M$ в [1] двухпетлевых вкладах $\Gamma_{4}$ есть легко исправимые опечатки), поэтому мы не будем здесь останавливаться на процедуре расчета. Его результаты для базовых функций $\Gamma_{2,4}$ в обсуждаемом сейчас простейшем приближении и без учета не существенных для дальнейшего (см. выше) УФ-конечных вкладов типа $A$ можно представить в виде

$$
\begin{aligned}
& \Gamma_{2}=i \hat{p}\left[1+\frac{1}{\varepsilon} t^{2 \varepsilon} \sum_{m s} u_{m} u_{s} T^{m s}+\ldots\right] \\
& \Gamma_{4}=\mu^{-2 \varepsilon} \sum_{k} \gamma_{A}^{(k)} \otimes \gamma_{A}^{(k)}\left[u_{k}+\frac{1}{\varepsilon} t^{\varepsilon} \sum_{m s} u_{m} u_{s} T_{k}^{m s}\right]+\ldots
\end{aligned}
$$

где $t \equiv c\left(p^{2} / \mu^{2}\right)$ с некоторой не существенной для дальнейшего нормировочной константой $c$ и

$$
\begin{aligned}
T^{m s} & \equiv \frac{1}{8}\left[-n \delta_{m s} G_{m}+H_{m s}\right] \\
T_{k}^{m s} & \equiv \frac{1}{2}\left[-n \delta_{k m} \delta_{k s} A_{k}+\delta_{k m} B_{k}^{s}+\delta_{k s} B_{k}^{m}-E_{k}^{m s}\right],
\end{aligned}
$$

с $n$ из (5). Входящие сюда величины $A, B, E, G, H$ - регулярные по $\varepsilon$ коэффициенты, которые порождаются различными свертками $\gamma$-структур и определяются следующим образом (по повторяюшимся векторным индексам и мультииндексам типа $A$ в $(1)$ - сум-

2 Теоретическая и математическая физика, т. 107, № 1, 1996 г. 
мирование):

$$
\left\{\begin{array}{l}
\operatorname{tr}\left[\gamma_{i} \gamma_{A}^{(k)} \gamma_{i} \gamma_{B}^{(m)}\right]=A_{k} \delta_{k m} \delta_{\mathrm{AB}} \operatorname{tr} 1 \\
\gamma_{B}^{(m)} \gamma_{i} \gamma_{A}^{(k)} \gamma_{i} \gamma_{B}^{(m)}=B_{k}^{m} \gamma_{A}^{(k)} \\
\gamma_{B}^{(m)} \gamma_{i} \gamma_{C}^{(s)} \otimes\left[\gamma_{B}^{(m)} \gamma_{i} \gamma_{C}^{(s)}-\gamma_{C}^{(s)} \gamma_{i} \gamma_{B}^{(m)}\right]=\sum_{k} E_{k}^{m s} \gamma_{A}^{(k)} \otimes \gamma_{A}^{(k)} \\
\operatorname{tr}\left[\gamma_{i} \gamma_{A}^{(m)} \gamma_{k} \gamma_{B}^{(m)}\right] \gamma_{A}^{(m)} \gamma_{l} \gamma_{B}^{(m)} \mathcal{D}_{i k l}=\hat{\partial} G_{m} \operatorname{tr} 1 \\
\gamma_{A}^{(m)} \gamma_{i} \gamma_{B}^{(s)} \gamma_{k} \gamma_{A}^{(m)} \gamma_{l} \gamma_{B}^{(s)} \mathcal{D}_{i k l}=\hat{\partial} H_{m s} \\
\mathcal{D}_{i k l} \equiv \delta_{i k} \partial_{l}+\delta_{i l} \partial_{k}+\delta_{k l} \partial_{i}
\end{array}\right.
$$

Явные выражения для этих коэффициентов приводятся в приложении 1.

Ренормировка величин (12) в схеме MS осушествляется простым вычитанием полюса по $\varepsilon$, т.е. в обозначениях (6)

$$
\begin{aligned}
& \Gamma_{2 \mathrm{R}}^{\mathrm{MS}}=i \hat{p}\left[1+\frac{1}{\varepsilon} \sum_{m s} u_{m} u_{s}\left(t^{2 \varepsilon} T^{m s}-\bar{T}^{m s}\right)+\ldots\right] \\
& \Gamma_{4 \mathrm{R}}^{\mathrm{MS}}=\mu^{-2 \varepsilon} \sum_{k} \gamma_{A}^{(k)} \otimes \gamma_{A}^{(k)}\left[u_{k}+\frac{1}{\varepsilon} \sum_{m s} u_{m} u_{s}\left(t^{\varepsilon} T_{k}^{m s}-\bar{T}_{k}^{m s}\right)\right]+\ldots
\end{aligned}
$$

Это соответствует в (8) константам ренормировки

$$
\begin{gathered}
Z_{1}^{\mathrm{MS}}=1-\frac{1}{\varepsilon} \sum_{m s} u_{m} u_{s} \bar{T}^{m s}+\ldots, \\
u_{k} Z_{2 k}^{\mathrm{MS}}=u_{k}-\frac{1}{\varepsilon} \sum_{m s} u_{m} u_{s} \bar{T}_{k}^{m s}+\ldots,
\end{gathered}
$$

а в (10) - РГ-функциям

$$
\gamma_{\psi}^{\mathrm{MS}}=-2 \sum_{m s} u_{m} u_{s} \bar{T}^{m s}, \quad \beta_{k}^{\mathrm{MS}}=2 \varepsilon u_{k}+2 \sum_{m s} u_{m} u_{s} \bar{T}_{k}^{m s}
$$

Младшими конечными $\gamma$-структурами являются все вклады $(15 \mathrm{a})$ и слагаемые с $k=$ $a=0,1,2$ в (15б). Поэтому из определения (13), (14) величин $T$ и общего правила 1 следует, что

$$
\bar{T}^{m s}=0, \quad \bar{T}_{a}^{m s}=0 \text { при } m s \neq b c,
$$

т.е. если хотя бы один из индексов $m s$ старший (см. соглашение об индексах в начале этого раздела). Из (18) следует, что полюсы по $\varepsilon$ в младших структурах (15) порождаются лишь младшими зарядами и что только они входят в младшие РГ-функции $\gamma_{\psi}$ 
и $\beta_{a}$. Они уже вычислялись ранее $[1,4]$, но для удобства приведем ответы в наших обозначениях (5) (их легко получить из (17) и справочных формул приложения 1):

$$
\begin{aligned}
\gamma_{\psi}^{\mathrm{MS}} & =(n-1) u_{0}^{2}+2 n u_{1}^{2}+4(n-1) u_{2}^{2}-4 u_{0} u_{1}+4 u_{0} u_{2}-8 u_{1} u_{2} \\
& \left\{\begin{array}{l}
\beta_{0}^{\mathrm{MS}}=2 \varepsilon u_{0}-2(n-2) u_{0}^{2}+8 u_{0} u_{1}-8 u_{0} u_{2}-16 u_{1} u_{2} \\
\beta_{1}^{\mathrm{MS}}=2 \varepsilon u_{1}-8 u_{0} u_{2} \\
\beta_{2}^{\mathrm{MS}}=2 \varepsilon u_{2}-4(n-2) u_{2}^{2}-4 u_{0} u_{1}-4 u_{0} u_{2}+8 u_{1} u_{2}
\end{array}\right.
\end{aligned}
$$

Однопетлевые $\beta$-функции (17) старших зарядов $u_{\alpha}$ зависят уже от всех зарядов $u$, явные выражения для них можно получить из справочных формул приложения 1.

Как уже говорилось, в пределе $\varepsilon=0$ зависимость ренормированных функций от старших зарядов может содержаться лиш в выражениях (15), порождаемых полюсом по $\varepsilon$ импульсных интегралов. Из (15) и (18) следует, что при $\varepsilon=0$ старшие заряды содержатся лиш в слагаемых, имеющих структуру локальных контрчленов (const внутри квадратных скобок (15)). Поэтому в согласии с утверждением (4) старшие заряды можно устранить подходяшей УФ-конечной ренормировкой, а именно умножением на У $\Phi$-конечную константу $Z^{\prime}(u)\left(Z^{\prime}=Z_{\phi}^{\prime 2}\right.$ в обозначениях (4)) выражения (15а) и переопределением $u_{a} \rightarrow u_{a}^{\prime}(u)$ младших зарядов в (15б) (учет $Z^{\prime}$ в (15б) в данном порядке не требуется ). Обозначив

$$
Z^{\prime} \equiv Z_{\phi}^{\prime 2}=1+\Delta Z^{\prime}, \quad u_{a}^{\prime}=u_{a}+\Delta u_{a}^{\prime}, \quad u_{\alpha}^{\prime}=u_{\alpha},
$$

можно предложить несколько вариантов такой УФ-конечной ренормировки:

$$
\begin{gathered}
\Delta Z^{\prime}=-\sum_{m s \neq b c} u_{m} u_{s} \overline{\bar{T}}^{m s}, \quad \Delta u_{a}^{\prime}=\sum_{m s \neq b c} u_{m} u_{s} \overline{\bar{T}}_{a}^{m s}, \\
\Delta Z^{\prime}=-\sum_{m s} u_{m} u_{s} \overline{\bar{T}}^{m s}, \quad \Delta u_{a}^{\prime}=\sum_{m s} u_{m} u_{s} \overline{\bar{T}}_{a}^{m s} \\
\left\{\begin{array}{l}
\Delta Z^{\prime}=-\sum_{m s} u_{m} u_{s}\left(T^{m s}-\bar{T}^{m s}\right) / \varepsilon \\
\Delta u_{a}^{\prime}=\sum_{m s} u_{m} u_{s}\left(T_{a}^{m s}-\bar{T}_{a}^{m s}\right) / \varepsilon
\end{array}\right.
\end{gathered}
$$

Все они соответствуют утверждению (4), т.е. при $\varepsilon=0$ полностью устраняют из ренормированных функций Грина зависимость от старших зарядов $u_{\alpha}=u_{\alpha}^{\prime}$. Ренормировка (22а) при нулевых старших зарядах сводится к тождественному преобразованию. В этом смысле данный вариант "минимален": устраняется лишь то, что совершенно необходимо устранить для реализации утверждения (4). Эквивалентные при $\varepsilon=0$ варианты (22б) и (22в) даже в отсутствие старших зарядов содержат дополнительную УФ-конечную ренормировку поля и младших зарядов, а именно

$$
\begin{aligned}
Z^{\prime}= & Z_{\phi}^{\prime 2}=1+(n-1) u_{0}^{2} / 4+(3 n+4) u_{1}^{2} / 2+(7 n+3) u_{2}^{2}- \\
& -3 u_{0} u_{1}+7 u_{0} u_{2}-10 u_{1} u_{2}+\ldots, \\
u_{0}^{\prime}= & u_{0}-(n-2) u_{0}^{2}+8 u_{0} u_{1}-16 u_{0} u_{2}-24 u_{1} u_{2}+\ldots, \\
u_{1}^{\prime}= & u_{1}+n u_{1}^{2}-2 u_{0} u_{1}-8 u_{0} u_{2}-4 u_{1} u_{2}+\ldots \\
u_{2}^{\prime}= & u_{2}+(2 n-24) u_{2}^{2}+2 u_{0} u_{2}-8 u_{1} u_{2}+\ldots,
\end{aligned}
$$


где многоточием обозначаются добавки со старшими зарядами. Этот вариант УФ-конечной ренормировки выделен тем, что соответствуюший функционал $\Gamma_{\mathrm{R}}^{\prime}\left(\phi, u^{\prime}\right)$ в (4) при $\varepsilon=0$ сохраняет, видимо, все формальные свойства симметрии двумерной модели (это проверено в [1] для двухпетлевых $\beta$-функций).

\section{3. РГ-ФУНКЦИИ В СЛЕДУЮЩЕМ ПОРЯДКЕ}

Если справедливо утверждение (4), то после нужной конечной ренормировки новые младшие РГ-функции

$$
\gamma_{\psi}^{\prime}=\gamma_{\psi}+\tilde{\mathcal{D}}_{\mu} \ln Z_{\phi}^{\prime}, \quad \beta_{a}^{\prime}=\tilde{\mathcal{D}}_{\mu} u_{a}^{\prime}=\beta_{a}+\tilde{\mathcal{D}}_{\mu} \Delta u_{a}^{\prime}
$$

при $\varepsilon=0$ должны зависеть только от новых младших зарядов $u_{a}^{\prime}$. Низшим порядком для РГ-функций являются выражения (19), (20), при $\varepsilon=0$ в них можно считать $u_{a}=u_{a}^{\prime}$ (поправки - превьшение точности), и эти выражения действительно зависят только от младших зарядов. В данном разделе мы проверим сохранение этого свойства в следуюшем порядке (две петли в $\beta_{a}$ и три в $\gamma_{\psi}$ ) и обсудим его следствия для РГ-функший в схеме MS. Для $\beta$-функций это уже было фактически проделано в работе [1], поэтому здесь мы ограничимся лишь краткими пояснениями и приведем отсутствуюшие в [1] явные выражения. Для трехпетлевой аномальной размерности $\gamma_{\psi}$ модели (8) такой расчет вьполняется впервые, нужные сведения о вкладах трехпетлевых графиков $\Gamma_{2}$ суммированы в приложении 2.

Рассмотрим сначала РГ-функции схемы MS. В рассматриваемом порядке импульсный интеграл $D_{p}$ любой диаграммы вместе с контрчленами от всех ее расходяшихся подграфов содержит УФ-конечную часть (вклад “типа $A$ "), полюс первого (тип $B$ ) и полюс второго (тип $C$ ) порядков по $\varepsilon$. РГ-фуункции в схеме MS определяются только полюсами первого порядка по $\varepsilon$ в константах ренормировки $Z$, т.е. вкладами типа $B$ с нулевым приближением по $\varepsilon$ в $\gamma$-структурах $D_{\gamma}$ и вкладами типа $C$ от поправок порядка $\varepsilon$ в $D_{\gamma}$. Обозначим соответственно

$$
\left.\beta_{k}^{\mathrm{MS}}\right|_{2 \text { петли }}=\left.\beta_{k}^{(2)}\right|_{B}+\left.\beta_{k}^{(2)}\right|_{C},\left.\quad \gamma_{\psi}^{\mathrm{MS}}\right|_{\text {3 петли }}=\left.\gamma_{\psi}^{(3)}\right|_{B}+\left.\gamma_{\psi}^{(3)}\right|_{C}
$$

Явные выражения для вкладов типа $C$ в РГ-функциях можно получить после несложных, хотя и громоздких вычислений из приведенных в [1] данных о двухпетлевых диаграммах $\Gamma_{4}$ (мы их перепроверяли), а для $\gamma_{\psi}-$ из результатов расчета трехпетлевых диаграмм $\Gamma_{2}$, приведенных в приложении 2. Ответы можно представить следующим 
образом:

$$
\begin{aligned}
\left.\beta_{k}^{(2)}\right|_{C}= & -n \sum_{m}\left\{u_{k}^{2} u_{m}\left[A_{k} B_{k}^{m}\right]+u_{k} u_{m}^{2}\left[B_{k}^{m} A_{m}\right]\right\}+ \\
& +\sum_{m s}\left\{u_{k} u_{m} u_{s}\left(n\left[A_{k} E_{k}^{m s}\right]-\left[B_{k}^{m} B_{k}^{s}\right]+2\left[B_{k}^{m} B_{m}^{s}\right]\right)+\right. \\
& \left.+n u_{m}^{2} u_{s}\left[E_{k}^{m s} A_{m}\right]\right\}+\sum_{m s p}\left\{-u_{k} u_{m} u_{s}\left[B_{k}^{p} E_{p}^{m s}\right]+\right. \\
& \left.+u_{m} u_{s} u_{p}\left(-2\left[E_{k}^{m s} B_{m}^{p}\right]-\left[B_{k}^{p} E_{k}^{m s}\right]+\sum_{l}\left[E_{k}^{l s} E_{l}^{m p}\right]\right)\right\}, \\
\left.\gamma_{\psi}^{(3)}\right|_{C}=- & \frac{1}{16}\left\{2 n^{2} \sum_{k} u_{k}^{3}\left[G_{k} A_{k}\right]^{\prime}-2 n \sum_{k m} u_{k}^{2} u_{m}\left(2\left[G_{k} B_{k}^{m}\right]^{\prime}+\left[H_{k m} A_{k}\right]^{\prime}\right)+\right. \\
& \left.+\sum_{k m s} u_{k} u_{m} u_{s}\left(4\left[H_{k s} B_{s}^{m}\right]^{\prime}+2 n\left[G_{s} E_{s}^{k m}\right]-2 \sum_{l}\left[H_{l s} E_{l}^{k m}\right]^{\prime}\right)\right\}
\end{aligned}
$$

где $A-H$ - введенные в (14) величины и для сокрашения записи использовано обозначение ( см. (6))

$$
[X Y] \equiv \bar{X} \overline{\bar{Y}}-\bar{Y} \overline{\bar{X}}, \quad[X Y]^{\prime} \equiv 2 \bar{X} \overline{\bar{Y}}-\bar{Y} \overline{\bar{X}}
$$

Порождаемые первым полюсом по $\varepsilon$ в импульсных интегралах вклады типа $B$ в младших РГ-функциях в силу обшего правила 1 зависят только от младших зарядов $u_{a}$, и для проверки обсуждаемого свойства (исчезновение зависимости от старших зарядов в младших РГ-функциях (25) при $\varepsilon=0$ ) их явный вид значения не имеет. При вычислении новых младших РГ-функций (25) при $\varepsilon=0$ в рассматриваемом приближении нужно учитывать, во-первых, указанные в (25) добавки от конечной ренормировки (21), во-вторых, поправки, порождаемые переходом от $u$ к новому аргументу $u^{\prime}=u+\Delta u^{\prime}$ во вкладах низшего порядка $(19),(20)$, что дает $(\varepsilon=0)$

$$
\left\{\begin{array}{l}
\beta_{a}^{\prime}\left(u^{\prime}\right)=\left.\left[\beta_{a}^{(1)}+\left.\beta_{a}^{(2)}\right|_{B}+\left.\beta_{a}^{(2)}\right|_{C}+L \Delta u_{a}^{\prime}-L^{\prime} \beta_{a}^{(1)}\right]\right|_{u=u^{\prime}} \\
\gamma_{\psi}^{\prime}\left(u^{\prime}\right)=\left.\left[\gamma_{\psi}^{(2)}+\left.\gamma_{\psi}^{(3)}\right|_{B}+\left.\gamma_{\psi}^{(3)}\right|_{C}+\frac{1}{2} L \Delta Z^{\prime}-L^{\prime} \gamma_{\psi}^{(2)}\right]\right|_{u=u^{\prime}} \\
L \equiv \sum_{k} \beta_{k}^{(1)} \partial_{k}, \quad L^{\prime} \equiv \sum_{b} \Delta u_{b}^{\prime} \partial_{b}, \quad \partial_{n} \equiv \partial / \partial u_{n}
\end{array}\right.
$$

Здесь $\beta_{a}^{(1)}$ и $\gamma_{\psi}^{(2)}$ - известные из (19), (20) младшие РГ-функции схемы MS при $\varepsilon=0$ (верхний индекс - число петель), предпоследние члены в правых частях (29) порождаются указанными в (25) добавками в $(21)$, а последние - заменой аргумента $u=u^{\prime}-\Delta u^{\prime}$ во вкладах низшего порядка.

Старшие заряды $u_{\alpha}=u_{\alpha}^{\prime}$ содержатся лишь в трех последних слагаемых (29). Используя соотношения $(27),(22),(13)$, можно проверить, что при выполнении конечной ренормировки по формулам (22б) или (22в) (эквивалентным при $\varepsilon=0$ ) три последних слагаемых в правых частях (29) полностью взаимно сокращаются (для $\beta$-функций это уже было показано ранее в [1]). Если же выполнять ренормировку по формулам (22a), обеспечивающим выполнение равенств $Z^{\prime}=1, u_{a}^{\prime}=u_{a}$ в отсутствие старших зарядов, то взаимное сокрашение трех последних вкладов будет неполным: от них останутся 
выражения (27) с суммированием только по младшим номерам во всех без исключения суммах, включая и четырехкратные (последнее уточнение не позволяет сказать, что в итоге получаются обычные выражения (27) схемы MS c нулевыми старшими зарядами, - в этом случае сумма по индексу "l" в (27) все равно оставалась бы полной). Отметим также, что для проверки высказанного выше утверждения нужны лишь выражения всех величин через определенные в (14) величины $A-H$, их явный вид не имеет значения.

Таким образом, любой из вариантов конечной ренормировки (22) приводит к устранению старших зарядов $u_{\alpha}^{\prime}=u_{\alpha}$ из РГ-функций (29) при $\varepsilon=0$. Наиболее простой вид эти РГ-функции принимают в “симметричной схеме” конечной ренормировки $(22 б$, в): тогда при $\varepsilon=0$

$$
\left\{\begin{array}{l}
\beta_{a}^{\prime}\left(u^{\prime}\right)=\left.\left[\beta_{a}^{(1)}(u)+\left.\beta_{a}^{(2)}(u)\right|_{\mathrm{B}}\right]\right|_{u=u^{\prime}} \\
\gamma_{\psi}^{\prime}\left(u^{\prime}\right)=\left.\left[\gamma_{\psi}^{(2)}(u)+\left.\gamma_{\psi}^{(3)}(u)\right|_{\mathrm{B}}\right]\right|_{u=u^{\prime}}
\end{array}\right.
$$

Во избежание недоразумений уточним, что запись $u=u^{\prime}$ в этих формулах подразумевает простую замену $u$ на $u^{\prime}$, а не выражение $u$ через $u^{\prime}$ по формулам (21), (22).

Вклады низшего порядка в (30) известны из (19), (20), явные выражения для двухпетлевых вкладов типа $B$ в младшие $\beta$-функции получены в работе [1] и в наших обозначениях (5) имеют вид

$$
\begin{aligned}
\left.\beta_{0}{ }^{(2)}\right|_{B}= & 4(n-2) u_{0}^{3}+8 n u_{0} u_{1}^{2}-16 u_{0}^{2} u_{1}+ \\
& +16 u_{0}^{2} u_{2}+16 n u_{0} u_{2}^{2}-16 n u_{1}^{2} u_{2}+64 u_{1} u_{2}^{2}, \\
\left.\beta_{1}{ }^{(2)}\right|_{B}= & 4(n-2) u_{0}^{2} u_{1}+16(n-2) u_{1} u_{2}^{2}- \\
& -16(n-2) u_{0} u_{1} u_{2}+16 u_{0}^{2} u_{2}+32 u_{0} u_{2}^{2}, \\
\left.\beta_{2}{ }^{(2)}\right|_{B}= & 16(n-2) u_{2}^{3}+4 n u_{0}^{2} u_{2}+16 u_{0} u_{2}^{2}+ \\
& +8 u_{0}^{2} u_{1}-32 u_{1} u_{2}^{2}+8 n u_{1}^{2} u_{2}-4 n u_{0} u_{1}^{2} .
\end{aligned}
$$

Мы вычислили также аналогичный трехпетлевой вклад в $\gamma_{\psi}$. Расчет довольно громоздкий и будет опубликован отдельно, а здесь мы приведем без вывода только конечный результат:

$$
\begin{aligned}
\left.\gamma_{\psi}^{(3)}(u)\right|_{B}= & (n-2)\left[(n-1) u_{0}^{3} / 2+4(n-1) u_{2}^{3}-3 u_{0}^{2} u_{1}+\right. \\
& \left.+3 u_{0}^{2} u_{2}+6 u_{2}^{2} u_{0}-12 u_{2}^{2} u_{1}+12 u_{0} u_{1} u_{2}\right]
\end{aligned}
$$

В заключение напомним, что зависимость от старших зарядов во вкладах типа $B$ в (26) отсутствует лишш в младших $\beta$-функциях $\beta_{a}$, а в старших $\beta_{\alpha}$ она сохраняется. Явные выражения для вкладов типа $B$ в $\beta_{\alpha}$ можно в принципе получить с помошњю предложенной в [5] техники вычисления $\gamma$-структур, в [5] приведены все нужные для двухпетлевого расчета справочные формулы. Но выкладки будут очень громоздкими, и пока они не проделаны. 


\section{4. ОБЩЕЕ ДОКАЗАТЕЛЬСТВО, ФОРМУЛЫ ПРОЕКЦИОННОЙ ТЕХНИКИ}

Сейчас мы приведем простое общее доказательство справедливости утверждения (4) во всех порядках теории возмущений. Фактически оно сводится к наблюдению, что для размерно-регуляризованной теории вместо обычной $R$-операции схемы MS можно ввести некоторую другую $R$-операцию, которая будет автоматически обеспечивать устранение зависимости от старших зарядов в ренормированных функциях Грина при $\varepsilon=0$.

Напомним сначала нужные для дальнейшего понятия и утверждения теории УФ-ренормировки (см., например, [6-8]). В ней используются операции $L, R, R^{\prime}$, действующие на диаграммы $D$ базовой теории, в нашем случае - модели (7). "Контрчленная операция" $L$ сопоставляет каждой поверхностно расходяшейся диаграмме $D$ соответствующий контрчлен $L D$ - некоторый полином по внешним импульсам нужной размерности. Через $L$ по известным комбинаторным правилам [6-8] строятся "неполная $R$-операция" $R^{\prime}=R^{\prime}(L)$, устраняющая все подрасходимости $D$, и “полная $R$-операция" $R=R^{\prime}-L$, устраняюшая все расходимости $D$.

Все эти операции, в свою очередь, рекуррентно выражаются соотношениями $L=$ $K R^{\prime}, R^{\prime}=R^{\prime}(L)$ через первичную “операцию выгитания" $K$ : она задается на функциях нужного типа и сопоставляет каждой из них ту ее часть, которую мы хотим устранить ренормировкой (например, только полюсы по $\varepsilon$ в схеме MS).

Основным аналитическим утверж дением теории ренормировки является следующее: если $L, R, R^{\prime}$ строятся через $K$ по стандартным правилам (что всегда предполагается) и если для всех диаграмм без подрасходимостей выбранная операция $K$ обеспечивает устранение УФ-расходимостей соответствующей $R$-операцией $R=1-K$ (на таких диаграммах $R^{\prime}=1$ ), то тогда для любой многопетлевой диаграммы $D$ расходимости величины $R^{\prime} D$ имеют такую же структуру, как и однопетлевые (простые полиномы по внешним импульсам нужной размерности). Поэтому они устраняются $R$-операцией т.е. величина $R D=\left(R^{\prime}-L\right) D=(1-K) R^{\prime} D$ расходимостей не имеет. Для дальнейшего важно подчеркнуть, что сформулированное утверждение фактически определяет структуру расходимостей любого “диаграммоподобного” многократного интеграла по импульсам, а не только конкретных выражений $D$, сопоставляемых диаграммам какой-нибудь квантово-полевой модели.

Главное здесь - гарантированная локальность (полиномиальность по внешним импульсам) расходимостей простейших однопетлевых интегралов (поскольку их расходяшиеся части заведомо исчезают при достаточном числе дифференцирований по внешним импульсам) плюс комбинаторное правило построения $R^{\prime}$-операции, вычитающей подрасходимости многократных интегралов. Поэтому можно утверждать, что все сказанное выше относительно "устройства УФ-расходимостей” относится не только к выражению $D=D_{\gamma} D_{p}$ для диаграммы в целом (см. п.2), но и к рассматриваемым отдельно импульсным интегралам $D_{p}$.

Что касается комбинаторики модели в целом, то основное утверждение можно сформулировать следующим образом [8]: ренормировка посредством применения $R$-операции к диаграммам базовой теории эквивалентна добавке к базовому действию контрчленов, определенных соотношением

$$
S_{\mathrm{R}}(\phi)=S_{\text {баз. }}(\phi)-L \Gamma_{\text {баз. }}(\phi),
$$


в котором $L-$ соответствуюшая контрчленная операция, а $\Gamma_{\text {баз. }}(\phi)-$ производящий функционал 1-неприводимых функций Грина базовой теории. Тем самым контрчлены действия представляются в виде суммы вкладов всех поверхностно расходящихся диаграмм с известными коэффициентами.

Вернемся теперь к нашей модели (7) с размерной регуляризацией. Обычная $R$-операция схемы MS определяется стандартной операцией вычитания $K \equiv K_{\mathrm{MS}}$ (отбор только полюсов по $\varepsilon$ ), применяемой к выражению $D=D_{\gamma} D_{p}$ для диаграммы в целом. Введем теперь новую операцию $\bar{K}$ и соответствующие $\bar{L}, \bar{R}, \bar{R}^{\prime}$, положив $\bar{K}\left[D_{\gamma} D_{p}\right]=D_{\gamma} K D_{p}, K \equiv K_{\mathrm{MS}}$. Это значит, что все новые ренормировочные операции действуют по обычным правилам схемы MS, но только на импульсные интегралы $D_{p}$, не затрагивая регулярных по $\varepsilon$ сомножителей $D_{\gamma}$, в частности

$$
\bar{L}\left[D_{\gamma} D_{p}\right]=D_{\gamma} L D_{p}, \quad \bar{R}\left[D_{\gamma} D_{p}\right]=D_{\gamma} R D_{p}
$$

Отметим что конечная ренормировка (22в) соответствует именно этой $\bar{R}$-операции в низшем порядке теории возмушений.

Покажем, что определенная таким образом $\bar{R}$-операция обладает всеми нужными свойствами. Из сказанного вьше относительно структуры расходимостей импульсных интегралов следует, что контрчлены $L D_{p}$ - полиномы по импульсам нужной размерности (первого порядка для $\Gamma_{2}$ и нулевого для $\left.\Gamma_{4}\right)$, а $R D_{p}-$ У $\Phi$-конечная величина. Поэтому в выражении $\bar{R}[D]=D_{\gamma} R D_{p}$ для диаграммы с $\bar{R}$-операцией можно прямо положить $\varepsilon=0$, в $\bar{R} D$ тогда останутся лиш младшие конечные $\gamma$-структуры (см. п.2), коэффициенты при которых не будут зависеть от старших зарядов в силу общего правила 1. Тем самым показано, что в ренормированных с помощью $\bar{R}$-операции функциях Грина зависимость от старших зарядов при $\varepsilon=0$ будет исчезать автоматически. Остается показать, что эти функции связаны с обычными ренормированными функциями схемы MS УФ-конечной мультипликативной ренормировкой типа (4). Для этого достаточно проверить, что действие $\bar{R}$-операции на диаграммы базовой теории также сводится к некоторой мультипликативной ренормировке той же неренормированной модели (3). Но это очевидно из свойств контрчленов $L D_{p}$ : для диаграмм $\Gamma_{4}$ это полиномы нулевого порядка по импульсам, т.е. некоторые выражения, составленные только из $\delta$-символов по векторным индексам. Поскольку в контрчлене $\bar{L} D=D_{\gamma} L D_{p}$ все векторные индексы должны быть свернуты, он обязательно сводится к линейной комбинации величин $\gamma_{A}^{(k)} \otimes \gamma_{A}^{(k)}$, т.е. вершин (2) в контрчленах действия (3). Очевидно также, что контрчлены любых диаграмм $\Gamma_{2}$ имеют вид const $\cdot \hat{p}$. Поскольку все контрчлены совпадают по структуре со слагаемыми (3), это доказывает мультипликативную ренормируемость базовой теории (7) с $\bar{R}$-операцией. При этом базовые (они же ренормированные) заряды этой теории, которые мы обозначим через $u^{\prime}$, следует отличать от аналогичных зарядов той же модели с обычной MS-ренормировкой, так как мы хотим, чтобы они соответствовали одной и той же неренормированной модели (3), а константы $Z$ в (9) для этих двух схем различаются. Связь между зарядами $u$ и $u^{\prime}$ определяется вытекаюшими из (9) соотношениями

$$
u_{0 k}=\mu^{-2 \varepsilon} u_{k} Z_{u_{k}}(u)=\mu^{-2 \varepsilon} u_{k}^{\prime} \bar{Z}_{u_{k}^{\prime}}\left(u^{\prime}\right)
$$


где $Z$ и $\bar{Z}$ - константы ренормировки для базовой теории типа (7) с $R$ и $\bar{R}$-операциями, соответственно.

Из сказанного следует, что функционал $\bar{R} \Gamma_{\mathrm{R}}\left(\phi, u^{\prime}\right)$ обладает всеми нужными свойствами и может играть роль $\Gamma_{\mathrm{R}}^{\prime}\left(\phi, u^{\prime}\right)$ в (4). Тем самым утверждение (4) доказано.

Функции $\Gamma_{n R}^{\prime}$ удовлетворяют РГ-уравнениям типа (11) с соответствуюшими РГ-функциями $\gamma_{\psi}^{\prime}\left(u^{\prime}\right), \beta_{k}^{\prime}\left(u^{\prime}\right)$. Их можно выразить с помощью РГ-уравнений не через константы ренормировки, а прямо через ренормированные функции Грина $\Gamma_{n R}^{\prime}$. В последних при $\varepsilon=0$ зависимость от старших зарядов исчезает, и это доказывает аналогичное свойство для младших РГ-функций $\gamma_{\psi}^{\prime}\left(u^{\prime}\right), \beta_{a}^{\prime}\left(u^{\prime}\right)$, остаюшихся в РГ-операторе при $\varepsilon=0$. Это свойство РГ-функций проверялось ранее (п. 2, 3) явно в двух первых порядках, и может показаться, что выкладки п. 2, 3 неоправданно громоздки по сравнению с приведенным выше простым доказательством. Но следует иметь в виду, что кроме проверки нужного свойства (исчезновение зависимости от старших зарядов в младших РГ-функциях при $\varepsilon=0$ ) там был выполнен конкретный расчет этих РГ-функций в двух порядках, и это самый простой метод вычисления. В этом отношении ситуация полностью аналогична проблеме УФ-конечности РГ-функций: обшее доказательство УФ-конечности сводится к простой ссылке на возможность выразить их через УФ-конечные ренормированные функции Грина с помощю уравнений РГ, но для конкретного вычисления всегда более удобны выражения РГ-функций через константы ренормировки $Z$, хотя они и содержат УФ-расходимости в виде полюсов по $\varepsilon$.

В заключение покажем, как из (4) можно получить формулы “проекционной техники" $[1,2]$, позволяюшие выразить при $\varepsilon=0$ старшие ренормированные составные операторы через младшие. Коротко говоря, искомые соотношения получаются дифференцированием равенства (4) по полям $\phi=\psi, \bar{\psi}$ и зарядам $u$. Чтобы пояснить это, напомним кратко основные определения теории ренормировки составных операторов [6-8].

Для любого построенного из классических полей $\phi$ и их производных локального монома $F \equiv F(x ; \phi)$ соответствуюший неренормированный составной оператор $\hat{F}(x)$ определяется соотношением $\hat{F}(x)=F(x ; \hat{\phi})$, в котором $\hat{\phi}$ - неренормированный квантовый оператор поля, а ренормированный $\hat{F}_{\mathrm{R}}(x)$ - соотношением $\hat{F}_{\mathrm{R}}(x) \equiv\left[F\left(x ; \hat{\phi}_{\mathrm{R}}\right)\right]_{\mathrm{R}}$, в котором $\hat{\phi}_{\mathrm{R}}=Z_{\phi}^{-1} \hat{\phi}$ - ренормированный оператор поля, а $[F(x ; \phi)]_{\mathrm{R}}-$ классический функционал - сумма исходного $F(x ; \phi)$ и операторных контрчленов. Последние являются некоторой линейной комбинацией всех мономов, которые могут "примешиваться" к исходному $F$ при ренормировке. В безмассовой теории таковыми могут быть лишь мономы, имеющие при $\varepsilon=0$ ту же самую каноническую размерность и те же свойства симметрии (в том числе и сам $F$ ). Коэффициенты при контрчленах содержат полюсы по $\varepsilon$, в схеме MS - только полюсы.

Обозначим для краткости через $\left\langle\left\langle\hat{F}_{\mathrm{R}}(x)\right\rangle\right\rangle$ производящий функционал 1-неприводимых функций Грина с одним оператором $\hat{F}_{\mathrm{R}}(x)$ и любым числом простых полей $\hat{\phi}_{\mathrm{R}}$. Такой функционал определяет оператор $\hat{F}_{\mathrm{R}}$ однозначно. Пусть $\Gamma \equiv \Gamma_{\mathrm{R}}^{\mathrm{MS}}(\phi, u)$ из (4). Из определения Г как преобразования Лежандра производяшего функционала связных функций Грина и представления последнего функциональным интегралом нетрудно показать (см.[8]), что для теории (8) с MS-ренормировкой (последнее важно)

$$
\frac{\partial \Gamma}{\partial u_{k}}=4 \pi \mu^{-2 \varepsilon} \int d x\left\langle\left\langle\hat{V}_{k R}(x)\right\rangle\right\rangle, \quad D_{\phi}(x) \Gamma=\left\langle\left\langle\hat{H}_{\mathrm{R}}(x)\right\rangle\right\rangle,
$$


где $\hat{V}_{k R}(x)$ - соответствуюший взаимодействию (2) ренормированный оператор, $D_{\phi}(x) \equiv$ $\phi(x) \frac{\delta}{\delta \phi(x)}\left(=\bar{\psi}(x) \frac{\vec{\delta}}{\delta \bar{\psi}(x)}+\frac{\overleftarrow{\delta}}{\delta \psi(x)} \psi(x)\right.$ для полей $\left.\phi=\psi, \bar{\psi}\right)$, а оператор $\hat{H}_{\mathrm{R}}(x)$ определен соотношением

$$
\begin{aligned}
\hat{H}_{\mathrm{R}}(x) & =\left.D_{\phi}(x) S_{\mathrm{R}}(\phi)\right|_{\phi=\hat{\phi}_{\mathrm{R}}}=\left.\left[D_{\phi}(x) S_{\text {баз }}(\phi)\right]_{\mathrm{R}}\right|_{\phi=\hat{\phi}_{\mathrm{R}}}= \\
& =\left.\left[\bar{\psi} \gamma_{i} \partial_{i} \psi-\partial_{i} \bar{\psi} \gamma_{i} \psi+16 \pi \mu^{-2 \varepsilon} \sum_{k} u_{k} V_{k}\right]_{\mathrm{R}}\right|_{\phi=\hat{\phi}_{\mathrm{R}}} .
\end{aligned}
$$

Отметим, что под знаком интеграла по $x$ два квадратичных по полям монома в (37) дают одинаковый вклад и что их разность $C$-четна, как и все $V_{k}$, тогда как их сумма $\partial_{i}\left[\bar{\psi} \gamma_{i} \psi\right]$ $C$-нечетна. Эти два монома плюс все $V_{k}$ образуют полную систему смешиваюшихся при ренормировке скалярных мономов с канонической размерностью $2+O(\varepsilon)$, причем к $V_{k}$ может примешиваться только $C$-четная разность из (37), а $C$-нечетный ток $\bar{\psi} \gamma_{i} \psi$ ренормируется мультипликативно.

Теперь мы можем явно сформулировать искомые соотношения. Рассмотрим равенство (4) непосредственно при $\varepsilon=0$, тогда $\Gamma_{\mathrm{R}}^{\prime}\left(\phi, u^{\prime}\right)$ зависит только от трех младших зарядов $u_{a}^{\prime}, a=0,1,2$. Перепишем равенство (4) компактно в виде $\Gamma=\Gamma^{\prime}$ с $\Gamma \equiv \Gamma_{\mathrm{R}}^{\mathrm{MS}}(\phi, u)$, $\Gamma^{\prime} \equiv \Gamma_{\mathrm{R}}^{\prime}\left(Z_{\phi}^{\prime-1} \phi, u^{\prime}\right)$ и продиффференцируем его по $u_{k}$ (напомним что $g=4 \pi u$ для всех зарядов). Учитьвая соотношения (36) и равенство $D_{\phi}(x) \Gamma=D_{\phi}(x) \Gamma^{\prime}$, получим бесконечную систему уравнений

$$
\int d x\left\langle\left\langle\hat{F}_{k R}(x)\right\rangle\right\rangle=\sum_{b} \frac{\partial u_{b}^{\prime}}{\partial u_{k}} \Gamma_{b}^{\prime}
$$

в которых $\Gamma_{b}^{\prime} \equiv \frac{\partial \Gamma^{\prime}}{\partial u_{b}^{\prime}}$ (частная производная без вкладов от $\left.Z^{\prime}\right)$ и

$$
\hat{F}_{k R}(x)=4 \pi \mu^{-2 \varepsilon} \hat{V}_{k R}(x)+\lambda_{k} \hat{H}_{R}(x), \quad \lambda_{k} \equiv \partial \ln Z_{\phi}^{\prime}(u) / \partial u_{k} .
$$

Из трех первых уравнений (38) для младших номеров $k=a=0,1,2$ три величины $\Gamma_{b}^{\prime}$ можно выразить через три младших оператора (39):

$$
\Gamma_{b}^{\prime}=\sum_{a}\left(M^{-1}\right)_{b a} \int d x\left\langle\left\langle\hat{F}_{a R}(x)\right\rangle\right\rangle, \quad M_{a b}=\partial u_{b}^{\prime} / \partial u_{a} .
$$

Подставив эти выражения в уравнения (38) для старших номеров $k \equiv \alpha>2$, получим аналогичные приведенным в [1] соотношения

$$
\int d x\left\langle\left\langle\hat{F}_{\alpha R}(x)\right\rangle\right\rangle=\sum_{a b} \frac{\partial u_{b}^{\prime}}{\partial u_{\alpha}}\left(M^{-1}\right)_{b a} \int d x\left\langle\left\langle\hat{F}_{a R}(x)\right\rangle\right\rangle
$$

выражаюшие интегралы по $x$ от старших $\hat{F}_{\alpha R}$ через младшие. При этом все коэффициенты в (41) выражены явно через (предполагающиеся известными) функции $Z_{\phi}^{\prime}(u)$, $u_{a}^{\prime}(u)$, характеризуюшие У $\Phi$-конечную ренормировку. 
На самом деле можно сделать еще один шаг, опустив в (41) знак интеграла по $x$. В обшем случае при этом могли бы появиться добавки примесей вида $\partial \hat{F}_{R}$ с неизвестными коэффициентами, вклады которых исчезают при интегрировании по $x$. В нашем случае такой опасности нет, так как единственной разрешенной по размерности примесью типа $\partial \hat{F}_{R}$ является дивергенция тока $\partial_{i}\left[\bar{\psi} \gamma_{i} \psi\right]$, но она запрешена по $C$-четности (см. выше).

Таким образом, при $\varepsilon=0$ все старшие ренормированные операторы $\hat{V}_{\alpha R}(x)$ можно выразить через три младших и $\hat{H}_{\mathrm{R}}(x)$ из (37).

Работа вьполнена при финансовой поддержке Шведской Королевской академии наук в рамках исследовательской программы Международного фонда фундаментальной физики в Москве (Д.С.Э. и К.Н.А.), Российского фонда фундаментальных исследований (проект № 93-02-14515) (В.А.Н.), Международного научного фонда и Российского правительства (грант $R$ 63300) (B.А.Н., К.Н.А.).

Приложение 1

\section{Коэффициенты $A-H$}

При вычислении введенных в (14) коэффициентов $A-H$ в произвольной размерности $d$ используется соотношение

$$
\operatorname{tr}\left[\gamma_{A}^{(k)} \gamma_{B}^{(m)}\right]=\operatorname{tr} 1 \varepsilon_{k} k ! \delta_{k m} \delta_{A B}, \quad \varepsilon_{k} \equiv(-1)^{\frac{k(k-1)}{2}},
$$

и вспомогательные величины $f, L, M$, определенные равенствами

$$
\begin{aligned}
\gamma_{B}^{(m)} \gamma_{\mathrm{A}}^{(k)} \gamma_{B}^{(m)} & =f_{k}^{m} \gamma_{\mathrm{A}}^{(k)}, \\
\gamma_{B}^{(m)} \gamma_{i} \gamma_{C}^{(s)} \otimes \gamma_{B}^{(m)} \gamma_{i} \gamma_{C}^{(s)} & =\sum_{k} L_{k}^{m s} \gamma_{\mathrm{A}}^{(k)} \otimes \gamma_{\mathrm{A}}^{(k)}, \\
\gamma_{B}^{(m)} \gamma_{i} \gamma_{C}^{(s)} \otimes \gamma_{C}^{(s)} \gamma_{i} \gamma_{B}^{(m)} & =\sum_{k} M_{k}^{m s} \gamma_{\mathrm{A}}^{(k)} \otimes \gamma_{\mathrm{A}}^{(k)}
\end{aligned}
$$

Явные выражения для них находятся [5] через производяшие функции от (одной или нескольких) вспомогательных переменных $u$ :

$$
\begin{aligned}
f_{k}^{m} & =\left.(-1)^{m k} \varepsilon_{k} \partial_{u}^{m}\left[(1+u)^{d-k}(1-u)^{k}\right]\right|_{u=0}, \\
L_{k}^{m s} & =\left.(-1)^{m s+m+s} C_{k}^{m s} \partial_{1}^{m} \partial_{2} \partial_{3}^{s}\left[a^{d-k} b^{k}\right]\right|_{u=0}, \\
M_{k}^{m s} & =\left.C_{k}^{m s} \partial_{1}^{m} \partial_{2} \partial_{3}^{s}\left[\tilde{a}^{d-k} \tilde{b}^{k}\right]\right|_{u=0},
\end{aligned}
$$

с $\varepsilon_{m}$ из (П1.1) (это четность перестановки $1 \ldots m \rightarrow m \ldots 1$ ) и $C_{k}^{m s} \equiv \varepsilon_{k} \varepsilon_{m} \varepsilon_{s} / k !, \partial_{i} \equiv$ $\partial / \partial u_{i}, a \equiv 1-u_{1} u_{2}-u_{1} u_{3}-u_{2} u_{3}, b \equiv u_{1}+u_{2}+u_{3}-u_{1} u_{2} u_{3}, \tilde{a} \equiv 1+u_{1} u_{2}+u_{1} u_{3}+u_{2} u_{3}$, $\tilde{b} \equiv u_{1}+u_{2}+u_{3}+u_{1} u_{2} u_{3}$.

Коэффициенты $L, M$ симметричны по перестановке верхних индексов $m, s$, отличны от нуля только при $k=m+s+1-2 l, l=0,1,2, \ldots$ и в таких обозначениях связаны 
соотношением $M_{k}^{m s}=L_{k}^{m s}(-1)^{m s+m+s+l}$ (доказывается заменой $u \rightarrow i u$ в определении $M$ ). Для этих коэффициентов из (П1.3) можно получить явное выражение (мы признательны С.С.Чабышевой, обратившей на это наше внимание)

$$
\begin{aligned}
L_{k}^{m s}= & \frac{m ! s ! \Gamma(d-k+1)}{l !(m-l+1) !(s-l+1) ! \Gamma(d-k-l+2)} \times \\
& \times[l(k+1)(d-k-p+1)+(d-k+1)(m-l+1)(s-l+1)],
\end{aligned}
$$

где $2 l=m+s+1-k, \Gamma(\ldots)$ - гамма-функция. Коэффициенты в (14) выражаются через величины из (П1.1)-(П1.3) следуюшим образом:

$$
\begin{gathered}
A_{k}=f_{k}^{1} \varepsilon_{k} k !, \quad B_{k}^{m}=f_{k}^{1} f_{k}^{m}, \quad E_{k}^{m s}=L_{k}^{m s}-M_{k}^{m s}, \\
H_{m s}=B_{s}^{m} f_{1}^{s}+B_{m}^{s} f_{1}^{m}+\sum_{k} L_{k}^{m s} f_{1}^{k}, \\
G_{m}=L_{1}^{m m}+M_{1}^{m m}+A_{m} f_{1}^{m} .
\end{gathered}
$$

Ниже приводятся явные выражения, которых достаточно для определения всех коэффициентов в $(14)$ с младшими $(\leq 2)$ номерами:

$$
\begin{gathered}
A_{k}=(-1)^{k} \varepsilon_{k} k !(d-2 k), \quad B_{k}^{0}=f_{k}^{1}=(-1)^{k}(d-2 k), \\
B_{k}^{1}=(d-2 k)^{2}, \quad B_{k}^{2}=(-1)^{k+1}(d-2 k)\left[d^{2}-d(4 k+1)+4 k^{2}\right], \\
L_{0}^{01}=M_{0}^{01}=d, \quad L_{0}^{12}=-M_{0}^{12}=2 d(d-1), \quad L_{1}^{00}=M_{1}^{00}=1, \\
L_{1}^{02}=-M_{1}^{02}=2(d-1), \quad L_{1}^{11}=M_{1}^{11}=3 d-2, \\
L_{1}^{22}=M_{1}^{22}=2(d-1)(5 d-8), \quad L_{2}^{01}=-M_{2}^{01}=1, \quad L_{2}^{12}=M_{2}^{12}=5 d-8, \\
E_{0}^{12}=4 d(d-1), \quad E_{1}^{02}=4(d-1), \quad E_{2}^{01}=2, \\
G_{0}=d+2, \quad G_{1}=d(d+2), \quad G_{2}=2 d(d+2)(d-1), \\
H_{00}=d+2, \quad H_{01}=d(d+2), \quad H_{02}=d(d+2)(1-d), \\
H_{11}=d(d+2)(2-d), \quad H_{12}=d(d+2)(d-1)(4-d), \\
H_{22}=d(d+2)(d-1)\left(d^{2}-9 d+16\right) .
\end{gathered}
$$

Для симметричных по $m \leftrightarrow s$ коэффициентов $L_{k}^{m s}, M_{k}^{m s}, E_{k}^{m s}, H_{m s}$ даны лишь значения для $m \leq s$. Все отсутствуюшие в приведенном списке коэффициенты с младшими $(\leq 2)$ номерами равны нулю.

Приложение 2

\section{Вклады второго полюса $1 / \varepsilon^{2}$ в трехпетлевые диаграммы $\Gamma_{2}$}

Выражение (12a) для $\Gamma_{2}$ с учетом трехпетлевых диаграмм можно представить в виде

$$
\Gamma_{2}=i \hat{p}\left[1+\frac{1}{\varepsilon} t^{2 \varepsilon} T^{\prime \prime}+\frac{1}{\varepsilon^{2}} t^{3 \varepsilon} T^{\prime \prime \prime}+\ldots\right],
$$


где $T^{\prime \prime}$ - вклады двухпетлевых диаграмм, $T^{\prime \prime \prime}$ - трехпетлевых. Для проверки сокращения вкладов старших зарядов в $\gamma_{\psi}^{\prime}$ и расчета величины (22б) нужны лишь те слагаемые, которые порождаются вкладами второго полюса $1 / \varepsilon^{2}$ импульсных интегралов. Эти данные в обозначениях (14) и приводятся ниже для всех девяти трехпетлевых диаграмм, пронумерованных в порядке следования на рисунке: от № 1 до № 9 (аналогичные данные для двухпетлевых диаграмм $\Gamma_{4}$ можно найти в [1]). Симметрийные коэффициенты и множители $-n=-N \operatorname{tr} 1$ от замкнутых циклов указываются отдельно внутри квадратной скобки, ответ приводится для величины $24 T^{\prime \prime \prime}$ (т.е. $T^{\prime \prime \prime}=\frac{1}{24} \times$ приведенное значение):

$$
\begin{aligned}
& \text { № } 1=\left[n^{2}\right] 2 \sum_{k} u_{k}^{3} G_{k} A_{k}, \\
& \text { № } 2=[-n] 2 \sum_{k m} u_{k}^{2} u_{m} G_{k} B_{k}^{m}, \\
& \text { № } 3=[-2 n] \sum_{k m} u_{k}^{2} u_{m}\left(G_{k} B_{k}^{m}+H_{k m} A_{k}\right), \\
& \text { № } 4+\text { № } 5+\text { № } 6=[1] 2 \sum_{k m s} u_{k} u_{m} u_{s}\left(H_{k s} B_{s}^{m}-\sum_{l} H_{l s} E_{l}^{k m}\right), \\
& \text { № } 7=[1] 2 \sum_{k m s} u_{k} u_{m} u_{s} H_{k s} B_{s}^{m}, \\
& \text { № } 8+\text { № } 9=[-n](-2) \sum_{k m s} u_{k} u_{m} u_{s} G_{s} E_{s}^{k m} \text {. }
\end{aligned}
$$

В эти выражения входят произведения $X Y$ с $X=G, H$ и $Y=A, B, E$, которые в (П2.1) умножаются на $t^{3 \varepsilon}$. При вычислении констант ренормировки $Z$ и ренормированных функций Грина необходимо учитывать в $T^{\prime \prime \prime}$ и вклады контрчленов от подграфов, которые также содержат полюсы $1 / \varepsilon^{2}$. Это легко сделать с помощью замены

$$
t^{3 \varepsilon} X Y \longrightarrow t^{3 \varepsilon} X Y-\frac{3}{2} t^{2 \varepsilon} X \bar{Y}=-\frac{1}{2} \bar{X} \bar{Y}+\frac{\varepsilon}{2}[X Y]^{\prime}+\ldots
$$

в обозначениях (6), (28). Вычитаемая в (П2.3) величина однозначно определяется обшей структурой контрчлена $\sim t^{2 \varepsilon} X \bar{Y}$ и необходимостью сокрашения $\ln t$ при полюсе $\sim 1 / \varepsilon$ (напомним что $t \sim p^{2} / \mu^{2}$ ). В аномальную размерность $\gamma_{\psi}^{\mathrm{MS}}$ дает вклад только второй член $\varepsilon$-разложения (П2.3), соответствуюший простому полюсу в константе ренормировки. Первый член порождает в $Z$ вклады $\sim 1 / \varepsilon^{2}$, которые должны быть определенным образом коррелированы с вкладами $\sim 1 / \varepsilon$ в диаграммах предыдушего порядка, что необходимо для сокрашения полюсов по $\varepsilon$ в $\gamma_{\psi}$. Мы проверили, что для приведенных выше данных нужные корреляции имеют место.

\section{Список литературы}

[1] Bondi A., Curci G., Paffuti G., Rossi P. // Ann. Phys. 1990. V. 199. №3. P. 268.

[2] Curci G., Paffuti G. // Nucl. Phys. B 1987. V. 286. P. 399.

[3] Kennedy A. // J. Math. Phys. 1981. T. 22. C. 1330; Авдеев A. // ТМФ. 1981. Т. 58. № 2. C. $308-314$. 
[4] Mitter P.K., Weisz P.H. // Phys. Rev. D 1973. V. 8. P. 4410.

[5] Васильев А.Н., Деркачев С.Э., Кивель Н.А. Препринт ПИЯФ ТН-42-1994, 1994.

[6] Коллинз Д. Перенормировка. М.: Мир, 1988.

[7] Завьялов О.И. Перенормированные диаграммы Фейнмана. М.: Наука, 1979.

[8] Васильев А.Н. // ТМФ. 1989. Т. 81. №3. С. 336.

Петербургский институт

Поступила в редакцию ядерной физики им. Б.П.Константинова 31.III. 1995 г Российской академии наук

\section{A.N. Vasil'ev, M.I. Vyazovskii, S.Ed. Derkachev, N.A. Kivel' \\ ON EQUIVALENCE OF RENORMALIZATIONS FOR STANDARD AND DIMENSIONAL REGULARIZATIONS OF $2 D$ FOUR-FERMION INTERACTIONS}

We discuss the problem of equivalence between the dimensional regularization in $d=2+\varepsilon$ and some other regularization in $d=2$ for the $U_{N}$-symmetric four fermion interactions. To make the multiplicative renormalization in $d=2+\varepsilon$ one need to introduce the infinite system of couplings $g \equiv\left\{g_{n}, n=0,1, \ldots\right\}$ but in $d=2$ there are three couplings only. We prove that after MS renormalization one can make UV-finite renormalization of couplings and field in such a way that after taking the limit of $\varepsilon=0$ all Green's functions will depend on three couplings only $g_{n}^{\prime}(g)$ with $n=0,1,2$. This gives the equivalence between the renormalizations in $d=2+\varepsilon$ and $d=2$. We calculate the two-loop $\beta$-functions and three-loop anomalous dimension of field in MS-scheme and give the rigorous proof of the so called "projection technique" which allows one to rewrite the higher renormalized operators through lower renormalized operators after the taking the limit of $\varepsilon=0$. 